\section{SELF ORGANISATION OF GOLD PARTICLES IN LANGMUIR}

\section{LAYERS}

M. J Everard ${ }^{1}$ C. L Nicklin ${ }^{1}$ M Brust ${ }^{2}$ S. Brown ${ }^{3}$ S. Baker ${ }^{1}$

${ }^{1}$ University of Leicester Physics and Astronomy University Road LEICESTER LEICESTERSHIRE LE1 7RH UK ${ }^{2}$ University of Liverpool, UK ${ }^{3}$ ESRF, Grenoble, France

The creation of ordered nanostructures is currently of great interest and has potential application for the design and fabrication of advanced optical, electronic, magnetic and sensor materials. A variety of techniques are available to form the self-organized structures on a solid surface, including the evaporation of a drop of solvent containing the nanoparticle clusters. An alternative method to create a more ordered layer is to allow the particles to assemble at the water/air interface, since it is perfectly flat and allows the nanoparticles to move more freely across its surface. The layer can then be transferred to a solid sample.

We present grazing incidence small angle $\mathrm{x}$-ray scattering (GISAXS) measurements from gold nanoparticle structures formed at the air-liquic interface in a langmuir trough and subsequently transferred to an ordered silicon substrate. The particles are produced using the two-phase method developed by Brust et al. The layer is formed, by allowing a drop of solvent containing the nanoparticle clusters to spread over a water surface. It is then transferred to the solid substrate using the Langmuir-Blodgett technique. The GISAXS measurements reveal the average particle size and the quality of the ordering as a function of cluster density, which is achieved by compressing the layer whilst on the langmuir trough. The results show that there is an optimal pressure range for the creation of two-dimensional structures and that there is some hysteresis in the structures formed due to irreversible agglomeration of the particles.

Keywords: SELF ASSEMBLY NANOPARTICLE LANGMUIR LAYER
Acta Cryst. (2002). A58 (Supplement), C163

OPTICAL SCHEME FOR SMALL ANGLE X-RAY SCATTERING BY USING MICROFOCUSING SOURCE AND 2D MULTILAYER OPTICS L. Jiang B. Verman B. Kim

Osmic, Inc. Department of Instrumentation 1900 Taylor Road AUBURN HILLS MICHIGAN 48326 USA

The most advanced laboratory small angle X-ray scattering (SAXS) systems use rotating anode, which offer higher flux and better spatially defined beams than sealed tube based systems. However, the beam from a rotating anode based system still has a large divergence. Thus a three-pinhole collimating system is often used to further define the beam to observe the scattering at a very small angle. In addition, the rotating anode based systems in general always have issues of high cost and high maintenance cost. The large divergence of the beam formed by a sealed tube or a rotating anode is caused by the large size of the source. The solution is using a microfocusing source that has a source size about 20 micrometers, and a closely coupled multilayer optic to take the advantage of its high brilliance. We have engineered an optical system based on this concept. For a compact system (about 2 meters), the performance a SAXS system based on this optical system is comparable to a rotating anode $(0.3 \mathrm{~mm}$ and $5 \mathrm{~kW})$ based system. Since the beam is better spatially defined and spectrally clean, a two-pinhole system composed of very thin pinholes is often adequate for many applications and offers much improved flux and resolution. Moreover, the systems with different resolution options and different lengths can be configured by using the same optical system and different pinhole systems. We will describe the design principles, optimization approaches and testing results.

\section{Keywords: MICROFOCUSING SOURCE MULTILAYER OPTICS PINHOLE CAMERA}

Acta Cryst. (2002). A58 (Supplement), C163

\section{SAXS AND WAXS CHARACTERIZATION OF NANOSTRUCTURED $\mathrm{CuO}$}

I. Torriani $^{1,2}$ A.E. Bianchi ${ }^{3}$ T. Plivelic ${ }^{2}$ S. Stewart ${ }^{3}$ G. Punte ${ }^{3}$

${ }^{1}$ Univ. Estadual De Campinas Condensed Matter Caixa Postal 6165 CAMPINAS SAO PAULO 13083-970 BRAZIL ${ }^{2}$ Laboratorio Nacional de Luz Sincrotron, Campinas, Brazil ${ }^{3}$ IFLP, Fac. de Ciencias Exactas, UNLP, Argentina

Particles with nanometric size generally exhibit properties significantly different from those of the bulk, and have recently acquired great importance because of their many applications in new materials development. In this work we present the results of the microstructural characterization of $\mathrm{CuO}$ produced by controlled ball milling (BM) and by solid state reaction (SSR). The nanocrystalline powders obtained from these methods are expected to be essentially different. The (BM) process induces highly deformed domains (strained crystallites separated by amorphous-like grain boundaries), whereas the (SSR) samples will contain nanocrystalline regions with sharp grain boundaries (with low dislocation density) after annealing. Strain and crystallite size have been calculated by Rietveld analysis of the diffraction patterns of all samples. SAXS results were used to describe the features of the interfaces and the volume fraction of the crystalline and the grain boundary phases based on a two-phase model for the systems. In the case of the BM samples, the behavior of the invariant indicates that the electron density contrast decreases as a function of milling time. Since an increase in the crystalline unit cell was verified from the XRD measurements, the results may indicate that ball-milling time increase causes a reduction of the crystalline phase density, with no change in the disordered phase. The (SSR) samples present different characteristics, with interfaces between the crystalline and grain boundary regions becoming smoother as a function of the thermal treatment. CAPES-SETCIP, CNPq, LNLS

Keywords: NANOSTRUCTURES SAXS COPPER OXIDE
Acta Cryst. (2002). A58 (Supplement), C163

DIMENSIONS OF PLECTONEMICALLY SUPERCOILED DNA Johan R. C. van der Maarel ${ }^{1}$ Wim Jesse ${ }^{1}$ Svetlana Zakharova ${ }^{1}$ Claude Backendorf $^{1}$ Stefan Egelhaaf ${ }^{2}$ Alain Lapp ${ }^{3}$

${ }^{1}$ Leiden University Leiden Institute of Chemistry PO Box 9502 LEIDEN 2300 RA NETHERLANDS ${ }^{2}$ University of Edinburgh ${ }^{3}$ Laboratoire Leon Brillouin

With a view to determine the configuration and regularity of plectonemically supercoiled DNA, we have measured the small angle neutron scattering (SANS) from pUC18 plasmid in saline solutions. Furthermore, we have derived the mathematical expression for the single chain scattering function (form factor) of a superhelical structure, including the longitudinal and transverse interference over the plectonemic pitch and radius, respectively. It was found that an interwound configuration describes the data well, provided interactions among supercoils are accounted for in the second virial approximation. The opening angle was observed to be relatively constant and close to 58 degrees, but it was necessary to include a significant distribution in radius and pitch. For diluted supercoils with vanishing mutual interaction, the derived structural results agree with independent measurements, including the distribution in linking number difference as determined by gel electrophoresis. With increasing plasmid concentration, prior and covering the transition to the liquid-crystalline phase, the radius and pitch are seen to decrease significantly. The latter observation shows that compaction of negatively supercoiled DNA by confinement results in a decrease in writhing number at the cost of a positive twist exerted on the DNA duplex. It is our conjecture that the free energy associated with this excess twist is of paramount importance in controlling the critical boundaries pertaining to the liquid-crystalline phase transition.

Keywords: SUPERCOILED DNA SCATTERING 